\title{
MERGING SIX EMERGENCY DEPARTMENTS INTO ONE: A SIMULATION APPROACH
}

\author{
Martin Miller \\ David Ferrin \\ Marshall Ashby \\ Tanner Flynn \\ FDI Simulation \\ 1707 East Highland, Suite 190 \\ Phoenix, AZ 85016, U.S.A.
}

\author{
Niloo Shahi \\ LAC+USC Healthcare Network \\ 1200 North State Street \\ Los Angeles, CA 90033, U.S.A.
}

\begin{abstract}
Simulation of existing systems can reinforce a Subject Matter Expert's gut feelings. However, it is more difficult to develop intuition for proposed systems, particularly when considering the consolidation of multiple systems. This paper discusses the use of simulation to determine the operational ramifications of combining six Emergency Departments into one of the largest in the country. Each of these six existing Emergency Departments serve a different type of patient population and each maintains their own independent processes. This hospital required all Emergency Departments to effectively function using the same floor space, processes and ancillary services, such as testing facilities, waiting rooms, and registration. Healthcare planners need to understand the ramifications of sharing resources among multiple departments and the operational impact of high volume systems. This project explored these challenges to find key bottlenecks and mitigation strategies using simulation.
\end{abstract}

\section{HOSPITAL OVERVIEW}

\subsection{Process Challenges}

The hospital was in the final stages of a 10 year, \$1 Billion construction to replace their 75 year old existing facility. As one of the largest teaching hospitals in the Southwestern United States, they operated six distinctly separate Emergency Departments with a combined 160,000 annual visits. The hospital knew they would face difficulties with the new facility. Although the square footage of the new Emergency Department (ED) is about 4 time larger than the combined areas, the number of beds is a few less than the sum of the beds in the six current Emergency Departments. Also, each of the existing Emergency Departments currently experience overcrowding. For example, patients in the Trauma Center today typically experience lengths of stay in excess of two days prior to admission or transfer.

Therefore, hospital management knew they must address their capacity constraints but were unsure of the effectiveness of their action plan. Also, they were unsure of the effectiveness of their ancillary support, such as radiology, lab, etc. Further, the new ED would need common processes and human resources, such as registration, patient transportation and nursing. Today, each ED operated with their own processes and people.

If the hospital can overcome these challenges, they would operate one of most efficient Emergency Departments in the country. Conversely, if the hospital opens without making the necessary changes, they could face a public relations nightmare.

\subsection{Simulation Advantages}

Simulation is one of the most widely used analytical techniques by professionals in Operations Research and Management Science (Law and Kelton 1991). Simulation analyzes the behavior of either real or imaginary systems over time and is usually performed on a computer using either off-the-shelf or customized software. The hospital decided to use simulation over other tools to address their challenges because simulation provides a highly effective technique for them to predict what will happen in the new facility [Miller, Ferrin and Messer 2004]. Simulation has been successfully used to model and analyze numerous emergency departments around the world (Mahapatra et al. 2003). Also, simulation allows the hospital to experiment with many scenarios without impacting the existing quality of patient care. (Miller, Ferrin and Szymanski 2003).

Using simulation results, hospital management can begin mitigating risks and solving issues months before they transition to the new facility. Examples of the kind of results ED simulation provided for previous hospitals include: 


\section{Miller, Ferrin, Ashby, Flynn and Shahi}

- Discharging inpatients about five hours earlier each day reduces ED patient LOS by a third,

- Adding 30 more inpatient beds will potentially cut the ED patient LOS in half,

- Reductions in lab test turnaround time won't significantly affect overall patient LOS until it is reduced by at least $20 \%$,

- The new ED only needs two-thirds of the proposed beds currently being designed (which will save millions of dollars),

- The new ED will handle up to 65,000 patients annually before ED LOS becomes unacceptable

\section{PROJECT APPROACH}

\subsection{Objectives}

Project management sought to provide a realistic simulation model which shows the capability of operational alternatives. The model would also show the associated value propositions for the ED and associated inpatient areas. The project would then analyze additional capacity requirements to support future volumes in the client's strategic plan. This capacity analysis includes:

- $3-5$ years volume impact projections

- ED capacity

- ED associated Inpatient capacity

\subsection{Modeling the ED}

The phases of a simulation project usually include

- Development of a conceptual model

- Programming the simulation and user interface software

- Testing the software

- Experimenting with specific scenarios,

- Presenting the results to project stakeholders.

FDI's approach to building a conceptual model involves facilitating several process modeling workshops, interviewing Subject Matter Experts (SMEs), and conducting process observations. The project team developed a conceptual model of the proposed, to-be processes of the ED. This required input from representatives of each of the six existing Emergency Departments. Together, the team easily reached consensus with one common process for ED patients to follow. Figure 1 shows a one page summary of this model. The process model encompasses more than just the ED because adjacent areas, such as inpatient floors, highly impact patient throughput.

The ED floor plan provided a starting point for activity order. For example, triage and registration areas are located between a single, main waiting room and smaller sub-waiting areas. This hospital originally planned for the patients to stay in the main waiting area until after triage and registration finish. However, the team was not constrained by the floor plans and developed alternative processes to test with the simulation model. The conceptual model was a useful interim deliverable because it allowed staff to visualize how the new patient processes would occur.

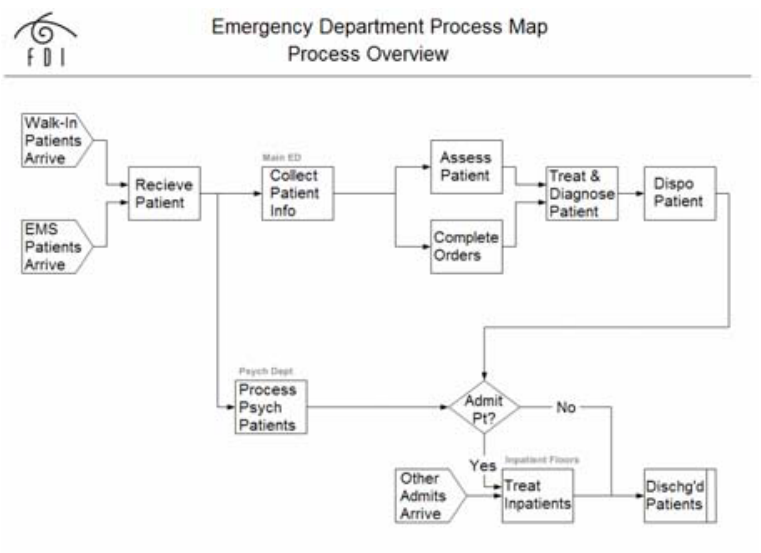

Figure 1: Conceptual Model of Emergency Department

\subsection{Data Collection}

The biggest challenge the team faced was collecting data from the six stand-alone Emergency Departments. The team collected activity duration estimates from Subject Matter Experts during the process modeling workshops. This provided an idea of the scope for each activity. It also provided a starting point for the team to focus their process observations. Those processes with the longest durations have the most impact on overall process performance. Therefore, it is critical that those processes are accurate.

Number of Guests/Patient

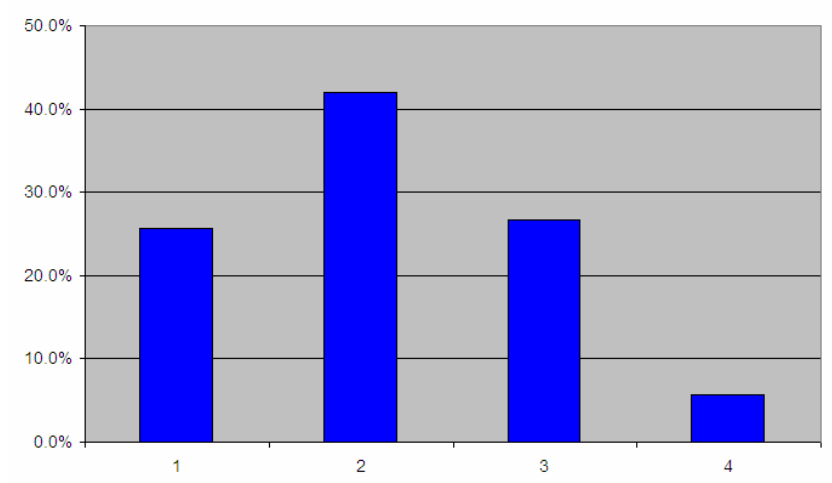

Figure 2: Sampled Number of Guests Per Patient

The team conducted many hours of observations, sampling data from each of the six Emergency Departments. Figure 2 shows an example of observed number of guests 


\section{Miller, Ferrin, Ashby, Flynn and Shahi}

that accompany each patient. It was not possible to observe all patients, so the team developed an effective plan to obtain sufficient data with the personnel available. The first objective from observations ensured the process, when applicable, actually occurs as the Subject Matter Experts described. The second objective gathered random samples of process data to create more accurate durations for the simulation model.

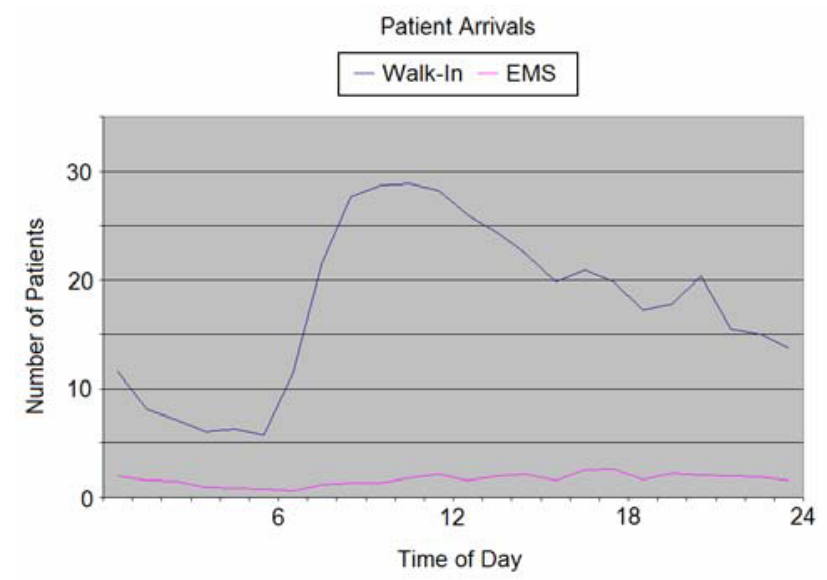

Figure 3: Walk-In and EMS Patient Arrivals by Time of Day

Electronic data from the hospital's Information Technology (IT) systems provided another key source of process data. Arrival data is usually the first data collected from hospitals because it is readily available. Hospitals usually track the time, location and mode of arrival for each of their patients (see Figure 3). Other forms of IT data include number of lab and radiology orders, turnaround time for order results, percentage of patients admitted, length of stay by patient type, etc.

\subsection{Software Development}

Constructing the simulation model and Graphical User Interface (GUI) each can occur simultaneously with data collection. FDI developers created a customized GUI which stores model parameters and facilitates creation and execution of alternative scenarios (see Figure 4). First, the team prepared a messaging architecture which shows the content and structure of model parameters. Then, developers concurrently built the GUI and simulation model following this architecture.

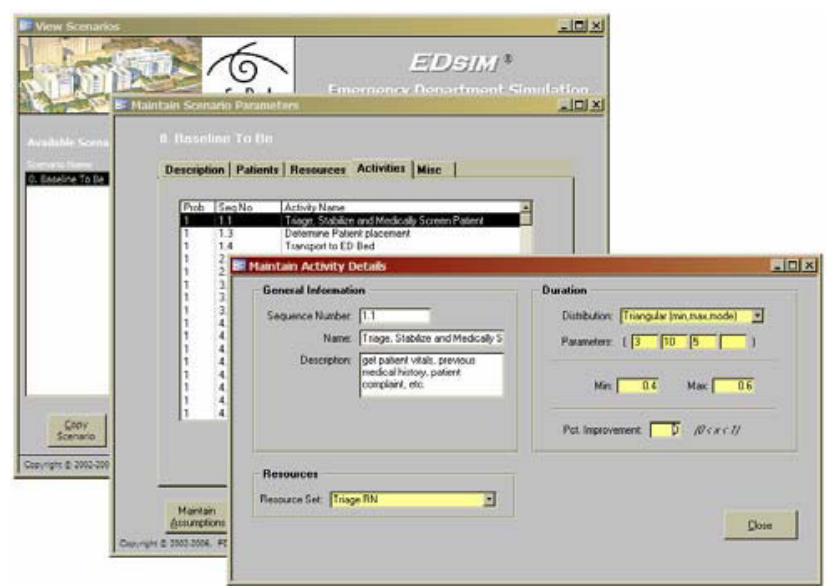

Figure 4: Customized Graphical User Interface

FDI developers followed a phased approach to building the simulation model. The developers unit test each phase of code before beginning the next phase of coding. The first phase of the model entailed generating entities in the right quantity and arrival pattern. The second phase involved routing entities to various locations using patient attributes or probabilities. Next, resources were added and activities coded which seized and released these resources for specified durations. The next phase includes coding key performance indicators such that model results are collected and exported to a spreadsheet for analysis. The final phase of simulation software development includes coding a compelling animation. FDI hired a third party vendor to convert the hospital's two dimensional CAD (Computer Aided Design) drawings to a three dimensional graphic (see Figure 5).

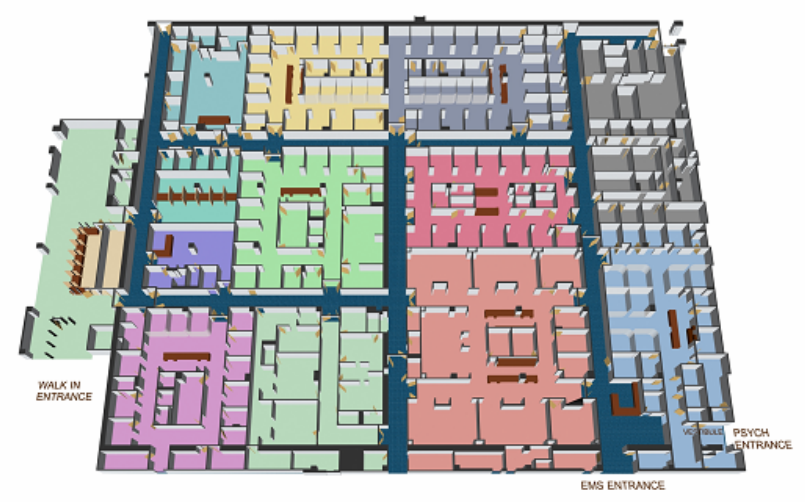

Figure 5: Background Graphic for ED Simulation

Once the GUI and simulation model were complete and fully tested, the software developers system tested the components together. The developers fixed all logic and performance issues that existed. Also, the developers finished entering newly collected data into the model. This project required additional time for data collection due to the scope of the ED system. The team compared simula- 


\section{Miller, Ferrin, Ashby, Flynn and Shahi}

tion results with current process results to ensure model validity (see Figure 6). Since this model represents the future ED and not the current six Emergency Departments, the team evaluated the simulation results appropriately.

\section{DTOD Simulation vs. Actual}

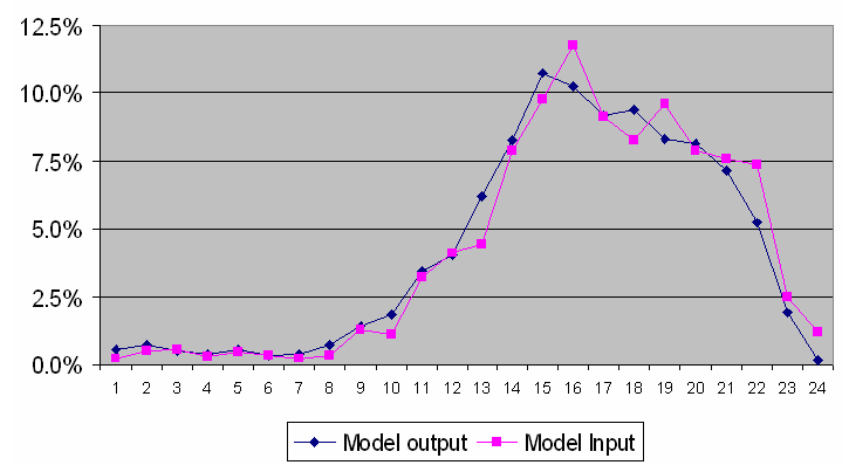

Figure 6: Example of Simulation Results Compared to Actual Values

\section{EXPERIMENTATION RESULTS}

Scenarios must be planned before experimentation runs can begin. The project team discussed scenarios with the client while establishing project goals and during the process modeling workshops. Simulation results quickly showed the lack of bed capacity, as expected. The average ED length of stay (LOS) went beyond initial expectations, though. Also, the simulation model unexpectedly showed major overcrowding in the main waiting room (see Figure 7). The hospital will not only combine six Emergency Departments but also combine six main waiting rooms.

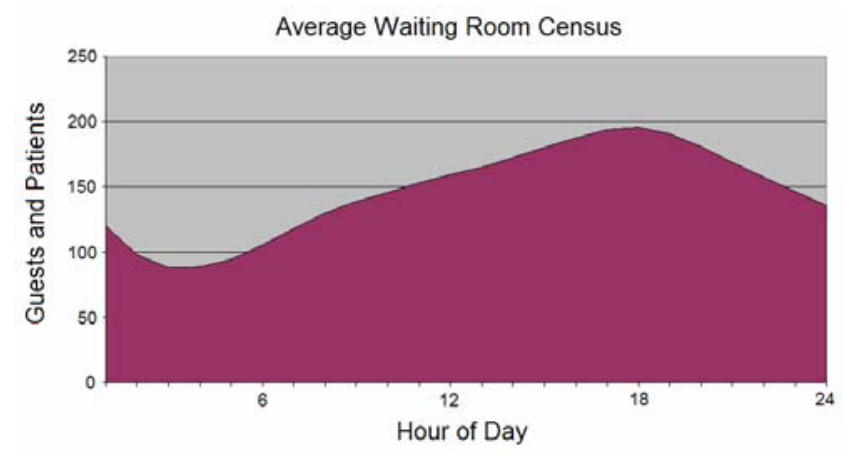

Figure 7: Expected Average Waiting Room Census

The team then setout to test scenarios which would improve patient cycle times to levels of the six current Emergency Departments. The first scenarios focused on adding bed capacity to determine how many beds would fix the unacceptable patient LOS. Simulation results showed the inpatient units were undersized by less than ten percent. The simulation model also showed additional ED beds would help, but only marginally. These additional beds might come in the form of a Transitional Stay Unit or extra Observations Unit beds. However, the hospital can not add more beds, so the team then focused on process improvement scenarios, such as:

- Bedside triage

- Bedside registration

- Reduce lab or radiology turnaround times

- Move the inpatient discharge time earlier in the day

- Streamline admitting activities

- Reduce inpatient length of stay by a half day

- Increase the inpatient occupancy rate to $95 \%$

Process improvements must be tested individually so that simulation results are attributed only to that particular modeling change. Once the best individual improvements were identified, the team began to combine scenarios to determine the best case scenario. Figure 8 shows the expected LOS distribution for several scenarios. Simulation results showed the average LOS will double if the hospital combines the six Emergency Departments into the new facility without making improvements. However, if the hospital implements the best case scenario, they could reduce the current LOS by at least $50 \%$.

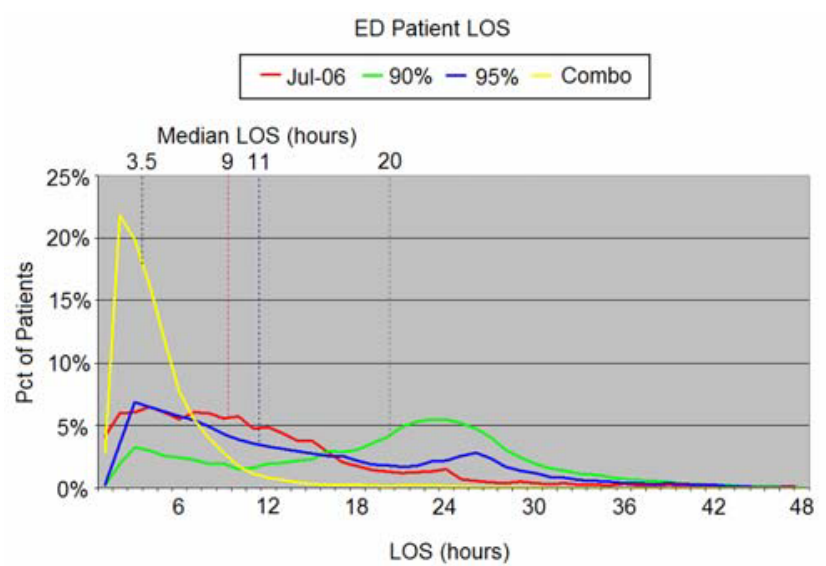

Figure 8: Expected ED Length of Stay for Alternative Scenarios

Hospital physicians wanted to know if they could hold open ED beds for specific types of cases, such as Trauma patients. The simulation model proved that even holding four beds for future events reduces capacity too far since the ED was already at full utilization.

\section{CONCLUSION}

Predicting the process performance of complex systems, such as Emergency Departments, is a challenging problem that can be best solved with simulation. Bottlenecks in the ED occur because patients arrive at a rate faster than they can be dispositioned. Determining how to eliminate the bottleneck is complex and usually involves testing many 


\section{Miller, Ferrin, Ashby, Flynn and Shahi}

scenarios. Combining six busy Emergency Departments into one presents more challenge with a wide range of outcomes. Simulation can not only show hospitals how to operate a new facility, but how to improve their patient throughput too. Hospital executives need to know where the major issues will occur so they can begin implementing a mitigation strategy.

\section{REFERENCES}

Law, A.M. and W.D. Kelton. 1991, Simulation Modeling and Analysis, McGraw-Hill, New York, NY.

Mahapatra, S., C.P. Koelling, L. Patvivatsiri, B. Fraticelli, D. Eitel, and L. Grove. 2003. Pairing Emergency Severity Index5-Level Triage Data with Computer Aided System Design to Improve Emergency Department Access and Throughput. In Proceedings of the 2003 Winter Simulation Conference, ed S. Chick, P. Sanchez, D. Ferrin, and D. Morrice, pp. 1917-1925, OMNIPRESS, Madison, WI.

Miller, M., D. Ferrin, and M. Messer. 2004. Fixing The Emergency Department: A Transformational journey with EDsim. In Proceedings of the 2004 Winter Simulation Conference, ed R. G. Ingalls, M. D. Rossetti, J. S. Smith, and B. A. Peters, pp. 1988-1993, OMNIPRESS, Madison, WI.

Miller, M., D. Ferrin, and J. Szymanski. 2003. Simulating Six Sigma Improvement Ideas For A Hospital Emergency Department. In Proceedings of the 2003 Winter Simulation Conference, ed S. Chick, P. Sanchez, D. Ferrin, and D. Morrice, pp. 1926-1929, OMNIPRESS, Madison, WI.

\section{AUTHOR BIOGRAPHIES}

MARTIN J. MILLER is a Senior Manager for FDI Healthcare Process Modeling. He previously worked over four years as a Senior Manger for Business Prototyping, Inc. developing simulation models and analysis primarily for the healthcare industry. He also worked over eight years for Accenture and was a Manager for their Capability Modeling and Simulation practice. He obtained his CMM for Software certification from the Software Engineering Institute in 1998. He received his Masters of Science in Industrial \& System Engineering and Bachelors of Science in Aerospace Engineering from the University of Florida. His email address is <mjmiller@fdiplan.com>

DAVID M. FERRIN is Principal for FDI Healthcare Process Modeling and has over 20 years of experience in Health Care consulting having worked with some of the largest and most prestigious health care systems in the nation serving on senior management teams, as department head and consulting. He was previously President of Business Prototyping, Inc. and an Associate Partner with Accenture's Capability Modeling and Simulation practice where he served on numerous Global Leadership Groups and as the Lead of the America's practice helping establish the world's largest international practice devoted to simulation. David has served as an Assistant Professor in the Health Systems Management department at Rush University, Chicago, Illinois and Adjunct Professor at York College of Pennsylvania. Mr. Ferrin is one of the most presented and published individuals in the nation in regards to simulation in health care. His email address is <dferrin@fdiplan.com>

MARSHALL W. ASHBY II is a consultant for FDI Healthcare Process Modeling. He also attends Arizona State University for continued education. He currently designs, builds, and analyzes discrete event simulations of new and existing healthcare facilities. He received his Bachelors of Science in Industrial \& System Engineering from Arizona State University and is currently pursuing a Masters in Health Sector Management and Six Sigma Black Belt, both from ASU. His email address is <mashby@fdiplan.com>

C. TANNER FLYNN is Associate Principal for FDI Healthcare Process Modeling. He brings over 15 years of professional experience in all levels of management in the banking industry as well as project management. He studied Sociology at Arizona State University. Prior to joining FDI, Tanner managed several large projects, including product development and technology conversions. Through his consulting business he managed hospital construction and relocation projects. His email address is <tflynn@fdiplan.com>

NILOO SHAHI is Assistant Hospital Administrator IV at LAC+USC Healthcare Network. She is in charge of process redesign projects related to the Move Transition activities for the Replacement Facility. She has over 18 years of experience in facility operations with various healthcare institutions mainly in the Los Angles County Public Hospitals. She has extensive experience in operational transformations, quality and process improvement in many departments within County operated facilities. She received her Master of Science in HealthCare Administration at California State University Northridge where as an Assistant Professor she also teaches Organizational Development in Healthcare. Her email address is <nshahi@lacusc.org> 